\title{
Weight Optimization of Syme Socket Prosthesis
}

\author{
Bakr Noori Khudher \\ Mechanical Department / College of Engineering \\ University of Mosul
}

\begin{abstract}
In this paper the finite element analysis was achieved on the Syme socket prosthesis to know the possibility of weight reduction of the socket. Previous works were restricted to stress analysis and socket-stump pressure distribution. In this work, the maximum stress induced in the socket model was found out at the maximum load carrying capacity that is developed at push-off stage of gait. The stress calculated is less than the yield and ultimate strength of the socket material, from which the optimum weight of the socket is obtained. The weight reduction of the socket is achieved by decreasing its thickness. At this point, the maximum stress is calculated again at the same loading condition as before reducing the weight. The maximum calculated stress is still less than the yield stress of the socket material. As a result, further weight reduction is achieved and hence the overall cost can be minimized by saving the expenditure on the material. Besides, the patients will feel comfort with light weight sockets.
\end{abstract}

Key Words: stress, Syme, socket, prosthesis, finite element, weight optimization.

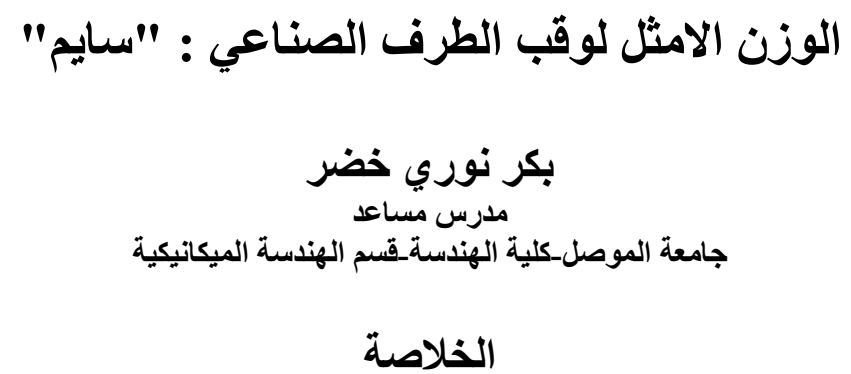

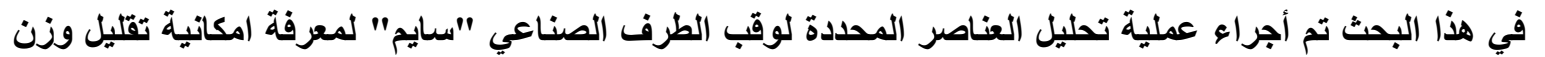

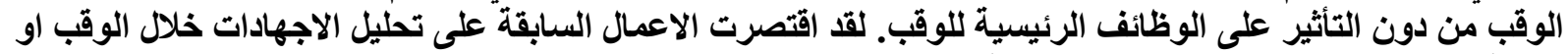

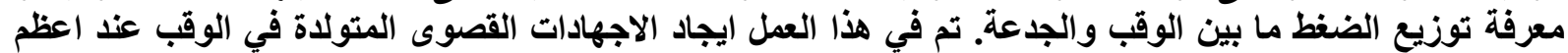

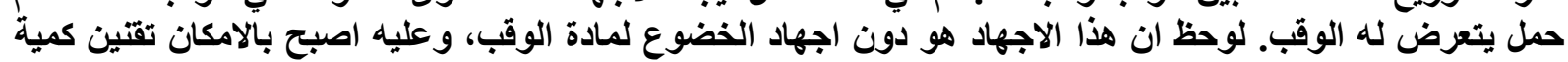

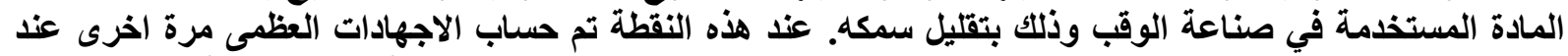

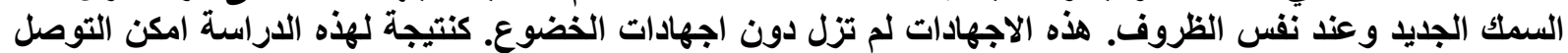

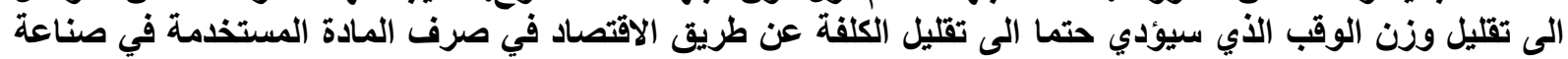

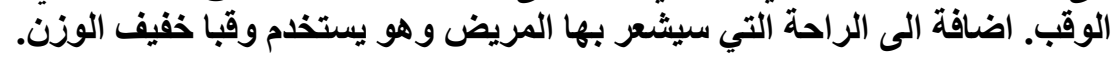

الكلمات المفتاحية: الاجهاد، سايم، وقب، العناصر المحدة، الوزن الامثل.

Received: 30 - 4 - 2012

Accepted: 11 - 9 - 2012 


\section{Introduction}

The amputation process can be defined as the removal, usually by surgery, of a limb, part or organ [1]. The purpose of amputation is to remove dead or diseased tissue, to relive pain, to obtain healing, and/or to rehabilitate the individual. Causes of amputation include trauma (loss of arterial supply, avulsion, and thermal injury), disease, and congenital deformity [2]. The level of amputation is selected based on the potential of healing and future function [3].

Syme amputation is a style of amputation named after Dr. James Syme, who developed the surgical teqnique in 1842. This type of amputation involves removing all bony elements of the foot while preserving the heel pad to facilitate weight bearing capacity [4] .

Following amputation, some type of artificial limb fitting had been practiced according to the type of amputation such as Syme prosthesis. Factories in some countries like Iraq supply the patients, with artificial limbs made of polypropylene sheets of thickness $4 \mathrm{~mm}$. This type of material is patient specific and doesn't create any problems like pressure sores even for diabetic patients. Though polypropylene is inexpensive, yet the factories are in need to cost reduction especially in developing countries. For that, This study is concerned with reduction of the manufacturing cost for Syme's socket prosthesis.

As the Syme socket been widely used, the socket's reliability, conformity and economic have received more and more attention. The finite element analysis is the main methods to test the economy and to optimize the design of the socket. Based on the basic theory of the finite element and force analysis, the socket is isolated with its free body diagram and the external forces are revealed at the dangerous main condition, push-off stage of gait. The finite element model is designed with the aid of ANSYS software package capabilities. The solution is achieved using two different thicknesses, the adopted and the proposed, by the recent version of ANSYS 12.1 software package.

Elangovan PT and et al [5] demonstrated a design synthesis analysis of the BK Syme prosthesis. They studied the cutout orientation size and shape, cutout fillet shape and socket wall thickness distribution.

Aladdin [6] made a study for socket strength enhancement, he made tests on the standard laminate and two proposed composite materials. He also fabricated three prototypes made of those three materials and tested under static load resembling the load condition at heel strike. Good enhancement strength achieved for socket materials throughout his proposed laminates.

Faustini [7], and his collogues have made analysis using finite element analysis of sockets manufactured by Selective Laser Sintering using Du- $(S L S)$ raform as the material. They tried to ensure reliability of the sockets for their use by patients and described the finite element models developed for the sockets, as well as the derivation of realistic boundary conditions that may allow a simulation of the structure under angular work loads.

This paper is a work of analysis and optimization using the stress result obtaining from the solution of Syme socket prosthesis using the finite element software package ANSYS 12.1. The overall manufacturing cost reduction is achieved by using the weight optimization model. The force calculations is achieved by using the biomechanical analysis at the worst stage of gait- push-off. The finite element analysis of the socket shell are performed for two different socket thicknesses.

\section{Syme Socket prosthesis Description}

The Syme prosthesis Fig. (1) consists of a socket, foot and liner. Syme socket also serves as a shank. Because of the short space between the end stump and the floor, a special type of foot, usually a modification of one of the popular designs, has to be used. Also, because of the shape of the stump, no extra provision of suspension is necessary. 
The plastic socket with soft insert liners are in two types: the plastic socket with posterior opening, and the plastic socket with medial opening that provide a mode of entry. Socket is a part of the prosthesis which accommodates the stump. Being a nonweight bearing prosthesis it may be either soft, firm or rigid. The socket may be made out of leather, plastic or metal. They may be metallic frames, single walled, doubled walled or silicon sleeves.

In this paper, Syme socket made of polypropylene with posterior cutout was chosen. The patient weight is $100 \mathrm{~kg}$. The material of the socket, adopted by Mosul Factory for Prosthetics and Orthotics, is polypropylene with mechanical properties; Young modulus is $1.17 \mathrm{GPa}$, Poisson's ratio is 0.33 , yield strength is 35 $\mathrm{MPa}$ and the density is $900 \mathrm{~kg} / \mathrm{m}^{3}{ }^{[8]}$. The measured thickness of the socket is $4 \mathrm{~mm}$.

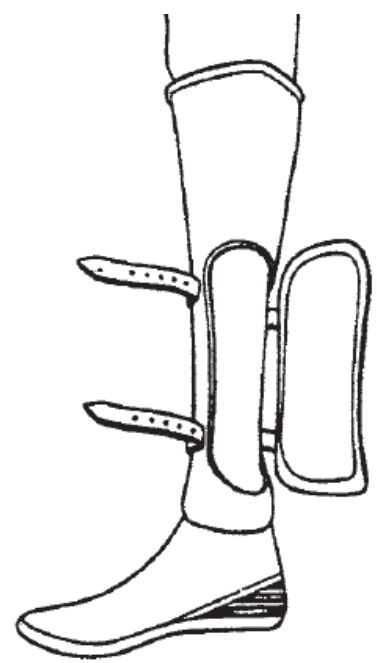

Figure (1) Syme

Prosthesis

\section{Biomechanical Force Analysis}

The gate cycle is a sequence of events starts from the heel strike on one foot to the next heel strike of the same foot. The cycle also subdivided into a stance and a swing phase. The stance phase comprises the heel strike, foot flat, push off, and toe off. The socket loading values vary according to the phase of gait. Obviously, the highest socket loading is developed at the push-off stage of gait ${ }^{[7]}$ and this phase is considered a design phase .

Fig. (2) shows the forces acting on the prosthesis. F represents the estimated floor reaction, $\mathrm{D}$ represents the corresponding stump force. Because $\mathrm{F}$ and the horizontal component of D don't intersect they cause the prosthesis to rotate counter clockwise on the stump. The moment is given by the term $F \times b$. This moment is countered by another force couple which produce a clockwise moment equals to $A \times a$, then this can be simply stated as $F \times b=A \times a$. For simplicity, the force $F$ is replaced by a force-couple system at the distal end of the stump. The estimated values of $a$ and $b$ are 40 and $15 \mathrm{~cm}$ respectively [8].

Examining the forces acting on the prosthesis in the sagittal plane immediately before push-off. The loading is idealized as an axial in-plane loading and a clockwise couple on the bottom edge of the circular cylinder socket shell, in addition to a couple produced by the anteroproximal and the posterodistal force.

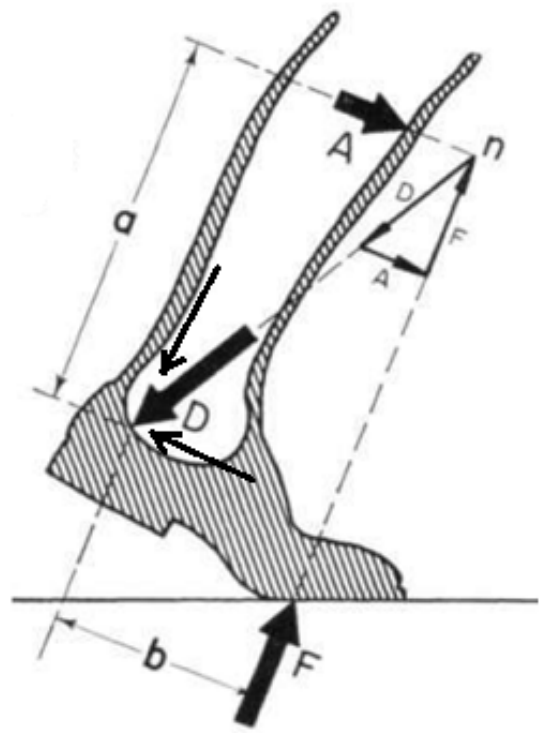

Figure (2) The prosthesis acting forces at the push-off stage of gait (Adopted from ref. 9)

\section{Solution Teqnique}

The shape modeling of the socket is created with the aid of software capabilities by dividing the socket into large numbers of stripes and measuring the coordinates of each stripes with respect to a fixed convenient origin. With ANSYS package software these points are created as well as the lines and areas Fig. (3).

In Syme socket, at push-off gait of stage, most of the body weight is transferred to the socket. By taking the physical subject weight, the ground reaction forces at the push-off stage 
of gait can be calculated. The lateral components for this study has been neglected as it is insignificant compared to the vertical forces.

The subject is $173 \mathrm{~cm}$ high and $100 \mathrm{~kg}$ weight. For the purpose of ANSYS procedure, the socket bottom edge is considered fixed and the loads are applied on the control nods at the anteroproximal and the distal posterior region of the socket (Fig. 5). The boundary condition for the socket concluded from the distal end of the socket attached to a fixed end. Thus all the nodes at the distal end with displacements and rotations are set to zero. Having all steps of the preprocessing are achieved including the selection of the element which is shell 63 and the mesh operation, the boundary conditions and loads are applied to obtain the solution. The post processing includes the plotting of contours where the stress distribution are found in different directions at the push-off stage of gait cycle.

$$
\begin{aligned}
& \text { Height of the socket }=27 \mathrm{~cm} \\
& \text { Max. diameter }=12.34 \mathrm{~cm} \\
& \text { Min. diameter }=9 \mathrm{~cm} \\
& \text { Material properties }{ }^{[8]} \text { : } \\
& E=1.17 \mathrm{GPa} \\
& v=0.33 \\
& \sigma_{\mathrm{y}}=35 \mathrm{MPa} \\
& \text { Density }=900 \mathrm{~kg} / \mathrm{m}^{3} \\
& \text { Thickness }=4 \mathrm{~mm}
\end{aligned}
$$

Figure (3) Shape modeling of the Syme socket

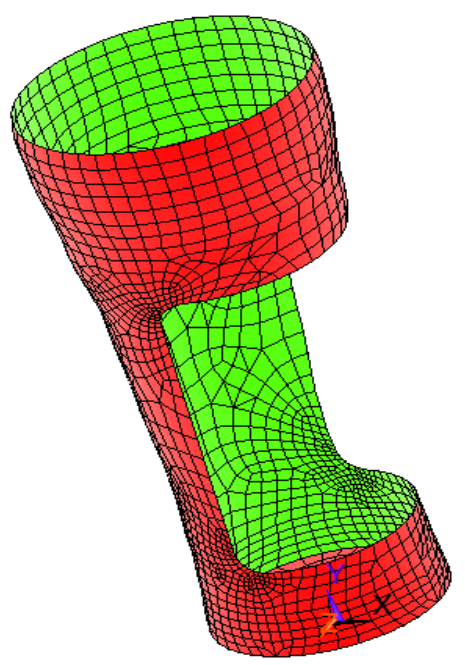

Figure (4) Finite element model
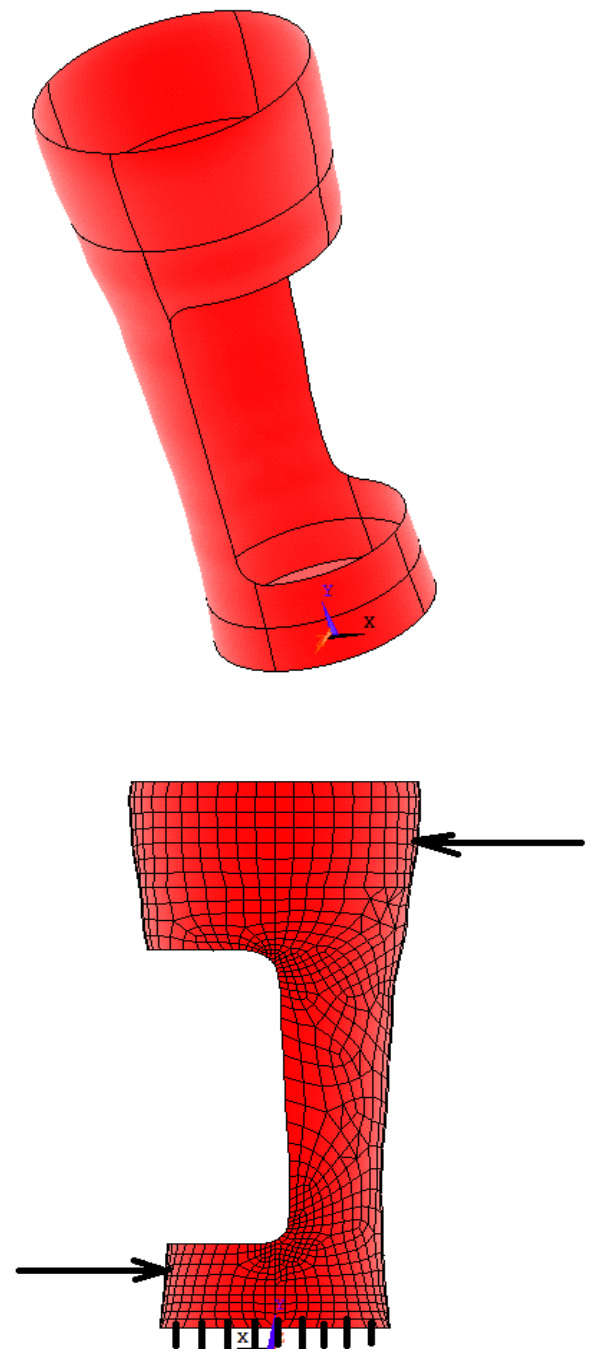

Figure (5) Socket free body

\section{Results}

The model optimized should perform intended functions and meet all requirements like stress level and keep the total weight to minimum. The thickness reducing is the main method to improve socket's economic, save materials and the cost of its manufacture. Through finite element models of the socket, stress distributions were obtained at the push-off stage of gait at the adopted thickness. Table (1) shows the maximum stresses developed in different orientations including Von Mises stresses obtained in the socket of tow thicknesses. 
Table (1) The maximum stresses developed in the sockets of 4 and $3 \mathrm{~mm}$ in MPa.

\begin{tabular}{|c|c|c|}
\hline Stress & $4 \mathrm{~mm}$ thickness & $3 \mathrm{~mm}$ thickness \\
\hline$\sigma_{x}$ & 2.28 & 3.48 \\
\hline$\sigma_{y}$ & -4.94 & -8.32 \\
\hline$\sigma_{z}$ & 5.75 & 9.89 \\
\hline$\sigma_{\text {VonMises }}$ & 5.36 & 9.18 \\
\hline
\end{tabular}

The stresses developed in the anterior-posterior (x), lateral-medial (y), and vertical $(\mathrm{z})$ in addition to the Von Mises stress are plotted via ANSYS software and shown in the Fig. (6) to Fig. (9) respectively.

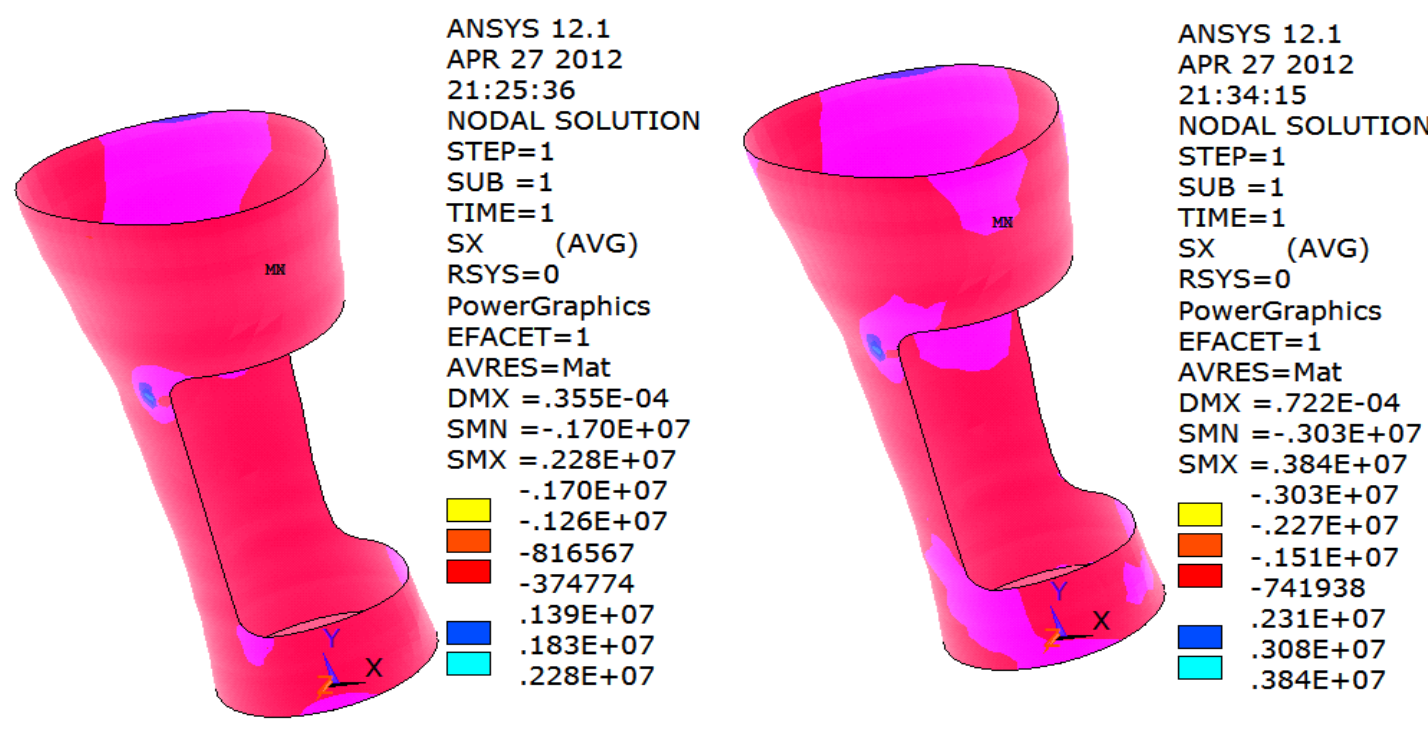

Figure (6) Contour plots of stress $\sigma_{\mathrm{x}}$ in the socket of $4 \mathrm{~mm}$ thickness (on the left) and $3 \mathrm{~mm}$ (on the right).
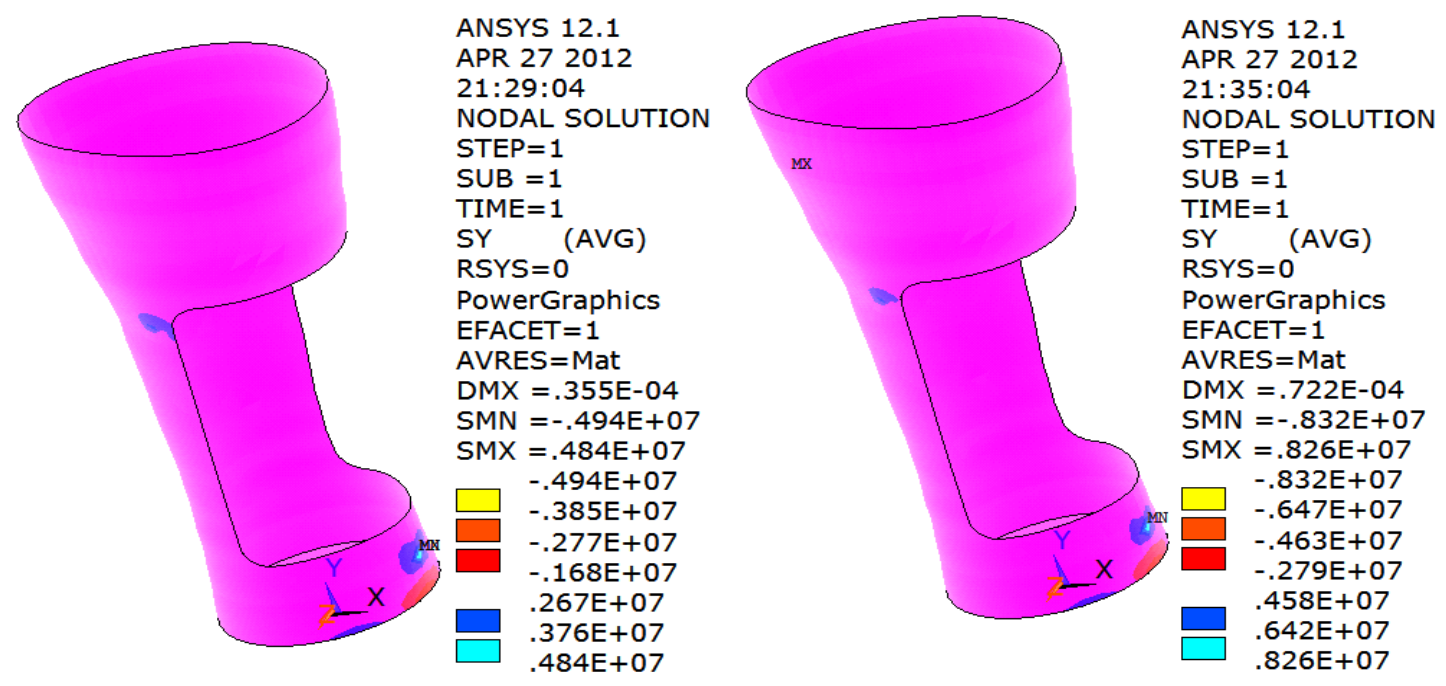

Figure (7) Contour plots of stress $\sigma_{\mathrm{y}}$ in the socket of $4 \mathrm{~mm}$ thickness (on the left) and $3 \mathrm{~mm}$ (on the right). 


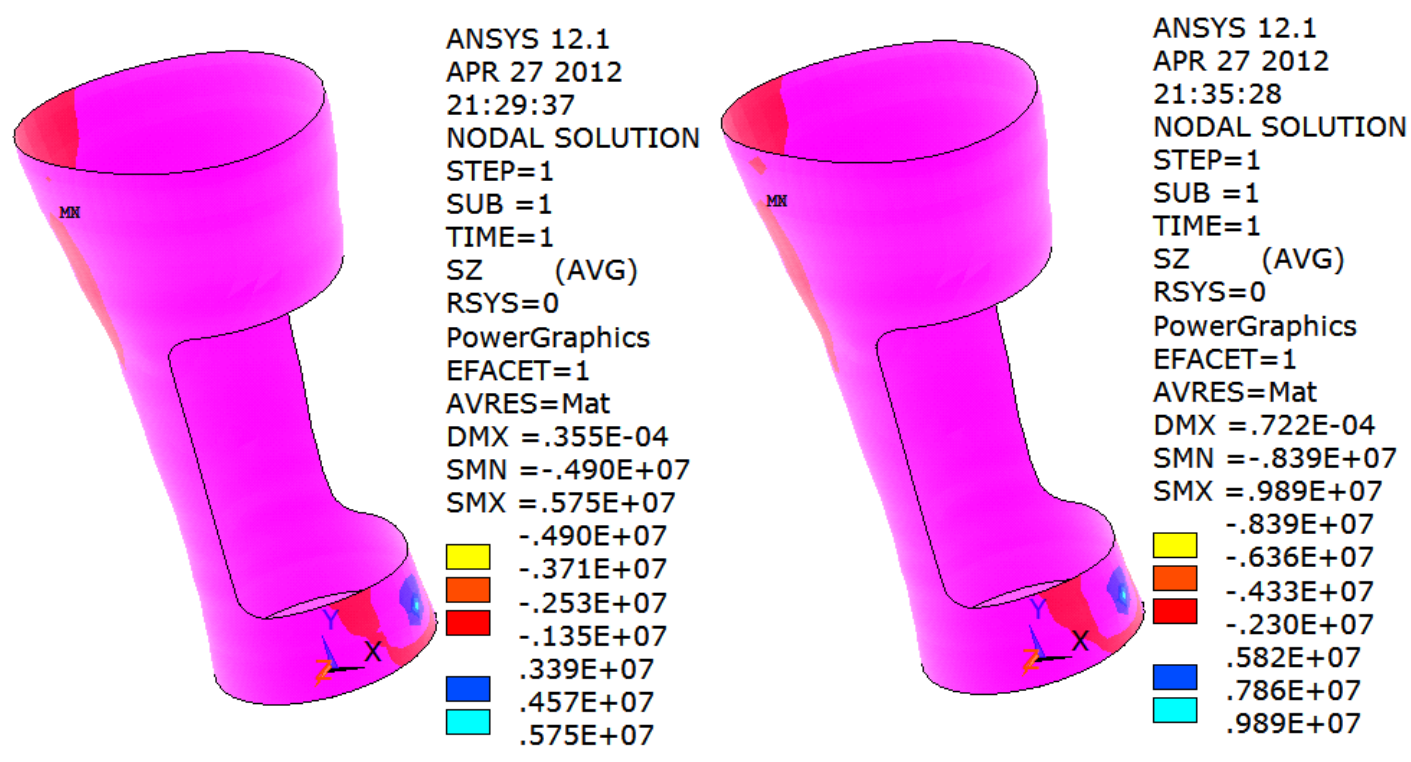

Figure (8) Contour plots of stress $\sigma_{\mathrm{z}}$ in the socket of $4 \mathrm{~mm}$ thickness (on the left) and $3 \mathrm{~mm}$ (on the right).

In Fig. (9), the maximum Von Mises stress in the new model of thickness $3 \mathrm{~mm}$ is 9.18 $\mathrm{MPa}$ which is greater than the stress developed in the old model of $4 \mathrm{~mm}$ during the push-off stage of gait. However, it is much less than the yield stress of the socket material, $35 \mathrm{MPa}$. Which means that the new model is also acceptable. This is confirmed also in the figures 6 to 9 as well
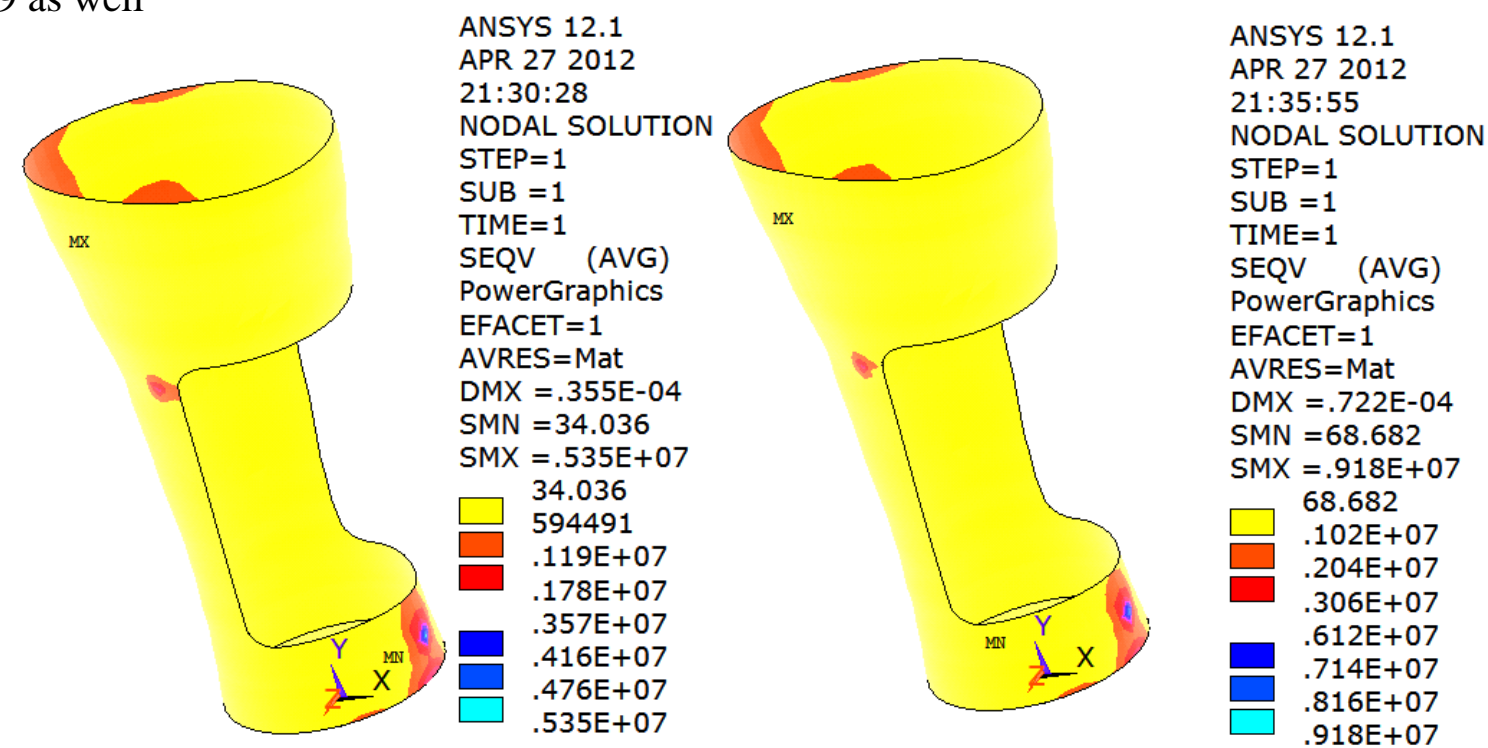

Figure (9) Contour plots of Von Mises stress in the socket of $4 \mathrm{~mm}$ thickness (on the left) and $3 \mathrm{~mm}$ (on the right).

\section{Discussion}

The weight optimization of the socket included only the thickness reduction and not for other aspects. This is because aspects such as shape cannot be changed as it is a mimic of the patient stump and according to individual differences. 
The contour plots of the stresses shoes how it concentrates at the lower edge of the socket. Even when the thickness is decreased, the stresses developed still much less than the yield stress. However, It cannot be decreased further as the deflection increases to high values.

Lighter socket weight is more comfortable for wearing, walking and other daily activities. This is achieved by reducing the thickness getting the socket weight is decreased by $25 \%$.

\section{Conclusions}

The following conclusions can be seen from this work:

1. In this study a further step is achieved with respect to other studies by reducing the weight with keeping the stresses in safe values.

2. The socket weight can be optimized successfully by decreasing the socket thickness.

3. The thickness cannot be decreased further even though the stress developed is much lower than the yield stress for the socket material to avoid large deflection.

4. The analysis of this study is under static loading. However, analyses under other conditions like jumping, running, up stair, etc. or fatigue loads are needed to sustain the results achieved.

\section{Glossary}

Anterior: In front of the body.

Distal: Furthermost part from the center of the body.

Gait: Manner of walking.

Lateral: Outside of the body.

Medial: Inside of the body on a lateral plane.

Phase: Particular instance of gait.

Posterior: Rear section of the body.

Prosthesis: An artificial substitution for a missing part of the human body.

Push-off: When the foot leaves the ground, to start the swing phase of gait.

Stance: Phase of gait when part of the foot is in contact with the ground.

Stump: Residual limb, the part that remains after amputation.

Swing: Phase of gait when the foot is off the ground.

Syme: A style of amputation occurs through ankle.

Socket: Component of prosthetic which contain residual limb.

\section{References}

1. Thomas, C.L. Taber's Cyclopedic Medical Dictionary. 17. Pheladelphia: F.A. Davis, 1993.

2. Shurr, D.G. and T.M. Cook. Prosthotics and Orthotics. Conn, East Norwalk: appelton and Lunge, 1990.

3. May, B.J. Amputations and Prosthotics; A Case Study approach. Philadelphia: F.A. Davis, 1996.

4.Mclaurin, C.A. The Syme type Prosthesis-Syme Amputation and Prosthesis.

5. Elongovan PT, Chista DN, Alwar RS. "Computer Design Synthesis of a BK Syme

Prosthesis." Mar, no. 9(2) (1979).

6. Aladdin, A. Development, and Testing of Syme Prosthesis with Enhanced Structural

Strength. Baghdad: Univercity of Technology, 2000, IRAQ.

7. Faustini, M., M. Lokandi, R. Grawford, W. Rogers, A. Gitter, and G. Boosker. "Finite

Element Structural Analysis of Prosthesis Socket for Below the Knee Amputees

Manufactured Using SLS." 2001: 381-392.

8. Muhsin J.J., and Bakr N.Alhasan. "Optimum Shape and Location of Opening in Syme Prosthesis Under General Loading." Al-Rafidain Engineering Journal, Vol. 16, No. 1, 2008, pp. 59. IRAQ.

The work was carried out at the college of Engineering. University of Mosul 